\title{
Desnutrición secundaria: impacto de las afecciones genéticas, metabólicas y neurológicas.
}

\author{
Drs. Y. Lacessie S.*, M. Colombo C.* e l. López S.*
}

\begin{abstract}
Fifty-nine merasinic infants hospitulized at the CDIP of LVTA because of difficulty in their nutritiongl and/or paychomotor recovery in the CCRN of CONIN, are analyzed. The cause of referral was lack of nutritional propress in 47.4\% of the cases, nutritional and psychomotor in 40.6 and only psychornotor in $11.8 \%$. The diagnosis made were: genetic, metabolic and neurologic diseages (HMN) in $42.4 \%$ : recurrent infections (R) in $35.5 \%$, gastrointestinal (GI) in 15.3\% and psychological $(P)$ in $6.8 \%$. 78.5\% of the children sent for a nutritional cause were diagnused as RI ur GI. When the failure of proeres was nutritional and psychomotor, $62.5 \%$ of the children were clasified as GMN. In the casth only dificulties in psychomotor recovery, 85.7\% corresponded to GMN.

It is concluded that the presence of severe psychomotor retardation is a gond index of $G M N$ pathulogy. Hecause of the heterogenity of the clinical diagnosis the anthroponetrical measures were of mo practical use in predicting the type of pathology.

The importance of sludying secondary malnutrition, especially in those cases where recovery fails, is emphazysed,
\end{abstract}

El crecimiento y desarrollo de un niño están determinados por la interacción de factores genéticos y ambientales. Una constitución genética adecuada es condición necesaria para el crecimiento y desartollo normal. Sin embargo, para la expresión total del potencial genético, es preciso satisfacer todos los requerimientos reales en forma proporcionada y continua.

En poblaciones con un elevado nivel de vida en que, generalmente, el ambiente satisface las necesidades, los factores genéticos contribuyen a explicar en mayor proporción las diferencias observables entre individuos (crecimiento, desarrollo, etc). Al mismo tiempo, la disminución de las patologias medio ambientales, produce un aumento relativo de las patologias de origen genético, adquiriendo esta relevancia como problema de salud.'

En países subdesarrollados, sectores importantes de la población se ven afectados por múltiples e intensas restricciones ambientales. Esta situación de privación constituye la principal determinante de la patologia prevalente $y$ de las diferencias observadas en crecimiento y desarrollo. ${ }^{2}$

En Chile prevalecen las patologias infecciosas y nutricionales, por lo cual la Pediatría y la Salud Pública se han orientado preferentemente a su tratamiento y prevención, dándole una primera prioridad a

-Unidad de Genética y Neuropaicología, Institnto de Nutrición y Tecnologia de los Alimentos, Universidad de Chile. la desnutrición infantil. Aunque la principal etiología es la falta de aporte de nutrientes (desnutrición primaria), ${ }^{3}$ existe una variedad de causas (hormonales, metabólicas, genéticas, etc.) que, interfiriendo el normal creciniento y degarrollo del niño, pueden originar secundariamente una desnutrición. ${ }^{4}{ }^{5}$ Cuando la desnutrición secundaria se presenta en niños de nivel socioeconómico alto ésta se sospecha precnzmente. No sucede lo mismo cuando se presenta en nivel socioeconómico bajo donde puede coexistir con una ingesta insuficiente, dando lugar a una desnutrición mixta.

La Corporación para la Nutrición Infantil (CONIN) tiene por objeto la rehabilitación integral (nutricional, psicomotora y social) de lactantes con desnutrición calórico-proteica severa primaria. ${ }^{6}$ Aquellos lactantes que no progresan en su recuperación nutricional y/o psícomotora en lós Centros de CONIN y cuya causa no ha podido establecerse a nivel local, son referidos para su estudio al Centro de Diagnóstico e Investigación Pediátrica (CDIP) del Instituto de Nutrición y Tecnología de los Alimentos (INTA) de la Universidad de Chile.

Los primeros casos estudiados demostraron la importancia de investigar sistemáticamente las causas secundarias de degnutrición en estos niños. El presente trabajo tiene como objetivos determinar e] impacto de esta patología, especialmente de las afecciones genéticas, metabólicas y neurológicas en este grupo e identificar variables que pudieran facilitar la detección precoz de estos casos. 


\section{MATERIAL Y METODO}

Se revisaron las histonias clínicas de los primeros 59 lactantes enviados para estudio al CDIP, consignando sexo, edad, causa de envio, mediciones antropométricas y evaluación del desarrollo psicomotor (DPM) al ingreso, y conclusión diagnóstica finalizado el estudio.

Los datos antropométricos se expresaron en porcentaje del promedio ideal para la edad, gegín Tablas del National Center for Health Statistics (N.C.H.S.). ${ }^{\text {? }}$ El DPM fue evaluado utilizando la Escala de Evaluación de Desarrollo Psicomotor (E.E.D.P. ${ }^{*}$ y expresado en un coeficiente de desarrollo (CD) cuyo promedio normal corresponde a 1.00 con una desviación standard de \pm 0.15 . En este estudio se consideró retraso leve entre -1 y -2 D.S., moderado entre -2 y -3 D.S. y severo bajo -3 D.S. del promedio.

Las conclusiones diagnósticas se agruparon en 4 categoriag:

1) Infecciones recurrentes (I.R.): incluye lactantes con historia de cuadros infecciosos a repetición, pero que presentan adecuada ganancia pondera] y avance en el DPM al estar libres de infecciones durante un período;

2) Gastrointestinales (G.I.): agrupa lactantes con patología digestiva demostrada (reflujo gastroesofágjeo, plicatura gástrica, fibrosis quística del páncreas, etc.). Este grupo no incluye diarreas infecciosas;

3) Psicológicas (Ps): aquellos lactantes sin patología orgánica comprobada, con retraso leve de au DPM, marcados antecedentes de privación afectiva y una respuesta adecuada al manejo conductual;

4) Genéticas, metabúlicas y neurológicas (G.M.N.): aquellos nìnos con trastornos neurológicos orgánicos, errores congénitos del metabolismo, alteraciones cromosómicas y síndromes con malformaciones congénitas que expliquen el retraso psicomotor y/o nutricional.

\section{RESULTADOS}

De los 59 lactantes estudiados, 35 eran de gexo magculino y 24 femenino.

En la Tabla N..$^{\circ}$ se observa la distribución de pacientes según causa de envío y categoría diagnóstica. En un $47,4 \%$ de los casos la causa de envio fue falta de progreso nutricional, en un $40,6 \%$ nutricional y psicomotora y exclusivamente psicomotora en un $11,8 \%$. En relación a categorias diagnósticas, $42,4 \%$ se incluye en enfermedades genéticas. metabólicas y neurológicas, $35.5 \%$ en infecciones recurrentes, $15,3 \%$ en gastrointestinales y $6.8 \%$ en psicológicas.

$$
\text { Tabla Nor }
$$

CIGTRBUCION ZE PACIENTES SEGIN CAUSAS DE ENYIO Y CATEGORIAS DIAGNCSTISA

\begin{tabular}{|c|c|c|c|c|c|}
\hline 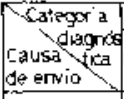 & Aectecciones & $\begin{array}{l}\text { Gastrs'nies } \\
\text { tiralos }\end{array}$ & Psisaligicas & $\begin{array}{l}\text { Gen éticas } \\
\text { vetabodicas } \\
\text { Nendxicicas }\end{array}$ & TOTAL \\
\hline Futricional & 15 & 7 & 2 & 4 & 28 \\
\hline $\mid \begin{array}{l}\text { N.גtriciorial } \\
\text { Ficomotora }\end{array}$ & 5 & 2 & I & $: 5$ & 24 \\
\hline Psincmotora & 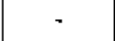 & - & 1 & 5 & 7 \\
\hline TOTAL & 21 & 9 & 4 & 25 & 59 \\
\hline
\end{tabular}

Tabla No2

CARACTERISTICAS ANTROPOMETRICAS Y COEFICIEN TE DE DESARROLLO (C.D.) SEGUN CAUSA DE ENVIO

\begin{tabular}{|c|c|c|c|}
\hline $\mathrm{N}$ & $\begin{array}{c}\text { Nutricional } \\
(28)\end{array}$ & $\begin{array}{c}\text { Nutricional } \\
\text { Psicomotora } \\
(24)\end{array}$ & $\begin{array}{c}\text { Psicomotora } \\
(7)\end{array}$ \\
\hline$\%$ PESO & 62.9 & 61.5 & 80.0 \\
\hline$\%$ TALLA & 86.7 & 86.1 & 88.0 \\
\hline$\%$ P/T & 84.9 & 86.3 & 99.6 \\
\hline$\%$ C.C. & 91.7 & 92.1 & 94.1 \\
\hline C. D. & $0.75^{* 0}$ & 0.48 & $0.48^{\circ}$ \\
\hline
\end{tabular}

$$
\begin{array}{rr}
* t=6.45 & 0 t=4.24 \\
p=<0.001 & p=<0.001
\end{array}
$$

En la Tabla N.* 2 se observan las caracteríaticas antropométricas y C.D. según causa de envío. Aquellos grupos en que la causa de envio incluye falta de progreso ponderal el déficit promedio de peso es superior al 37\% del peoo ideal para la edad, siendo sólo de un $20 \%$ para el gupo derivado exclusivamente por retraso del DPM. El déficit de talla es similar para los tres grupos, pero la relación P/T es normal en el grupo enviado sólo por retraso psicomotor. Los dos grupos con compromiso nutricional presentan un déficit $P / T$ entre un 14 y 15\%. El compromiso de circunferencia craneana (C.C.) es semejante en los tre grupos.

En lo relativo a C.D., en aquellos pacientes referidos por retraso psicomotor, el C.D. estaba bajo -3 D.S. (C.D. 0.55), mientras que en el grupo deriva- 
do por causa exclusivamente nutricional éste fue de 0.75 , lo que corresponde a un retraso leve.

Tabla №3

CARACTERIST:CAS ANTROPOMETRICAS Y COEFIC:ENTE DE DESARROLLO (C. D ) SEGUNN CATEGOALIAS DIAONNCSTICAS

\begin{tabular}{|c|c|c|c|c|}
\hline$N$ & $\begin{array}{c}1 \\
(21)\end{array}$ & $\begin{array}{r}G \mid \\
(9)\end{array}$ & $\begin{array}{l}\text { PS } \\
\{4\}\end{array}$ & $\begin{array}{l}G M N \\
(25)\end{array}$ \\
\hline$\%_{0}$ PESC & 618 & 65.3 & 73.7 & 945 \\
\hline \% TALLA & 85.9 & 8 B.3 & 87.5 & 865 \\
\hline$\%$ FIT & 86.0 & 84.2 & 335 & 862 \\
\hline$\%_{0} \subset C$. & 91.0 & 933 & $\subseteq 5.0$ & 32.8 \\
\hline C.D & 0.86 & 0.71 & पi 57 & 052 \\
\hline
\end{tabular}

Las características antropométricas y C.D. según categorías diagnósticas al ingreso al CDIP se observan en la Tabla N. ${ }^{\circ} 3$. Todos los grupos presentan un importante compromiso de peso, talla, P/T y C.C., excepto la categoría psicológica que muestra un déficit menor. El retraso psicomotor es gignificativamente mayor en la categoría genética, metabólica y neurológica ( $p<0.025$ ) que en las otras categorías, entre las cuales no hay diferencias $(p>0.50)$ ).

\section{DISCUSION}

Al analizar los resultados, en primer lugar llama la atención que el retraso en el DPM constituyó motivo de referencia en un $53 \%$ de los pacientes estudiados. Este retraso se caracteriza por ser más intengo y de más difícil recuperación que lo observado en lactantes desnutridos severos primarios tratados en los CCRN de CONIN, ${ }^{9}$ así como de aquellos lactantes enviados por causa nutricional exclusivamente (Tsbla N. ${ }^{a} 2$ ).

Cuando la falta de progreso es sólo nutricional, el $78,5 \%$ (22 casos) corresponde a las categorías de infecciones recurtentes y gastrointestinales. EJ DPM en estos casos está también comprometido, pero en forma similar a lo observado en el resto de los desnutridos. ${ }^{9}$ La principal causa de retraso psicomotor en estos grupos es la presencia de patología, como infecciones o reflujo gastroesofágico, que dificultan la estimulación psicomotora.

Si a la falta de progreso nutricional se agrega acentuado retraso psicomotor, un 62,5\% de los casos se califican como genéticos, metabólicos o neurológi- cos. Cuando la causa de envío es solamente retraso psicomotor, corresponde a la categoría genética, metabólica y neurológica, un $85,7 \%$ de los casos. Esto está claramente representado en el Gráfico N. ${ }^{\circ} 1$. De aqui que cuando hay sólo compromiso nutricional, la investigación de desnutrición secundaria debiera orientarse a causas gastrointestinales y más comúnmente a infecciones recurrentes, que no sólo disminuyen la ingesta, sino también significan aumento de los requerimientos. Cuando hay compromiso psicomotor acentuado, en primer lugar deben investigarse causas genéticas, metabólicas y neurológicas.

\section{Gráfico 1}

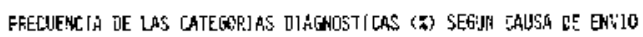

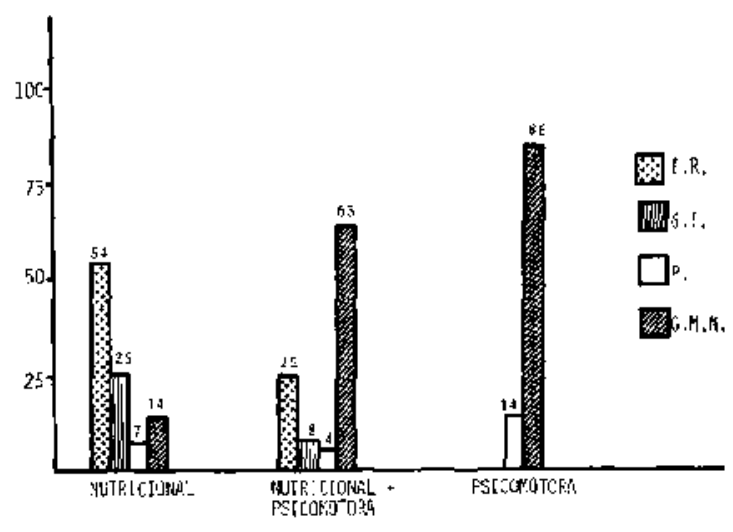

El análisis de los resultados obtenidos seriala que el DPM constituiría el elemento de detección de mayor importancia en el diagnóstico de patología genética, metabólica y neurológica. Dado que los desnutridos tienen habitualmente retraso psicomotor, con frecuencia resulta dif́́cil discriminar sólo con elementos clínicos aquellos casos más severos. De aquí la importancia de efectuar evaluaciones objetivas y sistemáticas del DPM que cuantifiquen el retraso, permitan valorar la evolución en relación al programa de estimulación y comparar con otras poblaciones.

Los indicadores antropométricos no resultaron orientadores de patología genética, metabólica y neurológica posiblemente por la gran heterogeneidad de la patología diagnosticada (Anexo 1 ).

El presente trabajo demuesira la importancis del pensar y buscar causas de desnutrición secundaria, en pacientes de nivel socioeconómico bajo, dado que las principales características de los desnutridos primarios: retraso de crecimiento y retraso psicomotor, son compartidos por muchas otras afecciones. Cree- 
mos que el hallazgo de patología secundaria, cuyo tratamiento no consiste únicamente en asegurar un adecuado aporte de nutrientes y estimulación psicomotora y afectiva explicaria, en gran medida, el 6-8\% de fracasos de recuperación observados en CONIN.

El determinar la causa precisa de la desnutrición reviste interés no sólo académico, sino también práctico, ya que permite en algunos casos un tratamiento etiológico y un pronóstico y consejo médico más adecuado.

\section{ANEXO 1}

\section{Diagnósticos efectuados}

\section{Infecciones recurrentes}

- Infecciones a repetición (infecciones urinarias, bronquitis, bronconeumonía, otitis, diarteas infeceiosas).

- Parasitosja

\section{Gastrointestinales}

- Insuficiencies gastroesofágicas

- Fibrosis quistica del páncreas

- Sindromes de mala absorción

- Plicatura gástrica

\section{Genéticas, metabolicas y neurológica}

- Síndrome XXY

- Síndrome de Silver-Russell

- Síndrome de Noonan

- Síndrome de Holt-Oram

- Síndrome Preudo-Rubinstein

- Síndrome de primer arco

- Sindrome de alcoholismo fetal

- Síndromes genéticos en identificación

- Anomalía de Klippel-Feil

- Fenilquetonuria

- Enfermedad orina jarabe de arce

- Mucopolizacaridosis

- Hipotiroidiomo

- Parálisis cerebral

- Dessnutrición intrauterina

- Craneosinostosis

- Miopatía

- Amaurosis congénita

\section{Psicologicas}

- Síndromes de privación afectiva

\section{RESUMEN}

Se analizan 59 nin̄os marásmicos, hospitalizados para estudio en el CDIP del INTA, por presentar dificultad en su recuperación nutricional y/o psicomotora en los CCRN de CONIN. La causa de envío fue falta de progreso nutricional en $47.4 \%$ de los cagos, nutricional y psicomotora en $\mathbf{4 0 . 6 \%}$ y exclusivamente psicomotora en $11.8 \%$. Las conclusiones diagnósticas correspondieron a: enfermedades genéticas, metabólicas y neurológicas (GMN) en un $\mathbf{4 2 . 4 \%}$; infecciones recurrentes (IR) en un $35.5 \%$; gastrointestinales (GI) en un $15.3 \%$ y psicológicas (P) en un 6.8\%. En un $78.5 \%$ de los nin̄os enviados por causa nutricional se encontró IR o afecciones GI. Cuando la falta de progreso era nutricional y psicomotora un $62.5 \%$ de los nin̈os se calificaron como GMN. En los casos en que sôlo había dificultad en la recuperación psicomotora, 85.7\% correspondía a GMN.

Se concluye que la presencia de retraso psicomotor severo es un buen indicador de patología GMN. Las características antropométricas no fueron de utilidad para predecir los diagnósticos, probablemente por la heterogeneidad de las afecciones estudiadas.

Se enfatiza la importancia de descartar una desnutrición secundaria en aquellos casos en que fracasa la recuperación.

\section{REFERENCIAS}

${ }^{1}$ Hall, J. G.; Powers, E. K.; Mcllvaine, R. T.; Ean V. H. The frecuency of genelic disense in a pediatric hospital. Am. J. Med. Genet. 1: 417, 1978.

2 Monckeberg, F. Jaque al Subdesarrollo. 3." Ed., Editora Nacional Gabriels Mistral L.tda., Santiago, 1976.

3 Monckeberg, F.; Donoso, G.; Valiente, S,; Arteaga, A.; Macctoni, A.; Merchak, N.; Oxman, S.; Lacassie, Y, Esindio del estado nutritivo y de las condiciones de rida de la pobleción infantil de la provincia de Curicó, Rer. Chit. Ped, 38: 512, 1967.

4 Beas, F.; Contreras, I.; Meccioni, A,; Lacassie, Y.; Monckeberg, $F_{\text {; }}$ Muzzo, S. Diqgánstico del retardo de crecimiento en el niño, Rev. Chil. Ped. B: 649, 1968.

${ }^{5}$ How Children Grow. Clinical Aesearch Advances in Human Geowth and Development. DHEw. Publications N.* (NIH) 73166,1973

6iumallo, J. A. Progrema de Centroa Cerrados de Recuperacion Nutricional en Chile. Simpoeio lnternacional de latervención Nutricional, Santiago, 19?8.

${ }^{7}$ National Center for Health Statistics Growth Charts. U.S.A. Department of Health Edtucation and Welfare. Healh Hosources Administration, 25: 76, 1976 .

${ }^{8}$ Rodriguez, S.; Arancibia, V, Undurraga, C. Eecale de Evaluar ción de Desarrollo Psicomotor : D-24 meses. Universidad Católica. Santiago, 1976.

${ }^{9}$ Colombo, $\boldsymbol{M}$; Lopez, I. Evolución del desarrullo pricomotor de lactantes desnutridos severos sometidos a un programa de rehabililación integral. Ped. Hes. 14, 1979 (Abetract). 